Tohoku J. Exp. Med., 2007, 211, 387-393

\title{
Plasma Interleukin-8 as a Potential Predictor of Mortality in Adult Patients with Severe Traumatic Brain Injury
}

\author{
Aleksandar Gopcevic, ${ }^{1}$ Branka Mazul-Sunko, ${ }^{1}$ Jasminka Marout, ${ }^{2}$ \\ Ante Sekulic, ${ }^{3}$ Natasa Antoljak, ${ }^{4}$ Mladen Siranovic, ${ }^{1}$ Zeljko Ivanec, ${ }^{1}$ \\ Marko Margaritoni, ${ }^{5}$ Miroslav Bekavac-Beslin ${ }^{6}$ and Neven Zarkovic ${ }^{7}$ \\ ${ }^{1}$ Department of Anesthesiology and Intensive Care, University Hospital Sestre Milosrdnice, \\ Zagreb, Croatia \\ ${ }^{2}$ Laboratory of Endocrinology, University Hospital Sestre Milosrdnice, Zagreb, Croatia \\ ${ }^{3}$ University Department of Anesthesiology, Zagreb, Division of Neuroanesthesia and \\ Neurointensive Care, University Hospital Zagreb, Croatia \\ ${ }^{4}$ Institute of Public Health, Zagreb, Croatia \\ ${ }^{5}$ General Hospital, University of Dubrovnik, Dubrovnik, Croatia \\ ${ }^{6}$ Department of Surgery, University Hospital Sestre Milosrdnice, Zagreb, Croatia \\ ${ }^{7}$ Department of Molecular Medicine, Rudjer Boskovic Institute, Zagreb, Croatia
}

Gopcevic, A., Mazul-Sunko, B., Marout, J., Sekulic, A., Antoljak, N., Siranovic, M., Ivanec, Z., Margaritoni, M., Bekavac-Beslin, M. and Zarkovic, N. Plasma Interleukin-8 as a Potential Predictor of Mortality in Adult Patients with Severe Traumatic Brain Injury. Tohoku J. Exp. Med., 2007, 211 (4), 387-393 — Because of complex pathophysiology and severe consequences, traumatic brain injuries (TBI) are an important medical problem. Pathophysiology of TBI includes local and systemic stress response, in which interleukin- 8 (IL-8) is considered as a key mediator of neuroinflammation. However, prognostic relevance of IL-8 measurement in adult patients with severe TBI is not certain. Therefore, IL-8 was determined in blood samples from central venous and jugular bulb catheter and in cerebrospinal fluid of twenty patients with isolated TBI at admission to Intensive Care Unit. None of the patients had history of stroke, dementia, autoimmune diseases, acute infection or medication with anti-inflammatory drugs. Ten patients died due to traumatic brain injury, while the other ten recovered well. While there was no significant difference of IL-8 levels in cerebrospinal fluid between survivors and nonsurvivors, central venous plasma level of IL-8 was significantly lower in survivors $(71.00 \pm 14.17 \mathrm{pg} / \mathrm{ml})$, than in nonsurvivors $(111.26 \pm 16.9 \mathrm{pg} / \mathrm{ml})$. Receiver Operating Characteristic (ROC) analysis revealed significant prognostic value for IL-8 in the blood as well as for the age of patients, Glasgow Coma Scale (GCS) and Acute Physiologic and Chronic Health Evaluation (APACHE II). These findings suggest that the central venous plasma values of IL-8 at admission might be an early predictive marker in patients with severe TBI, comparative to standard clinical prognostic markers such as APACHE II and GCS. — traumatic brain injury (TBI); coma; mortality; inflammation; Interleukin-8 (IL-8)

(C) 2007 Tohoku University Medical Press

Received January 30, 2007; revision accepted for publication March 5, 2007.

Correspondence: Neven Zarkovic, M.D., Ph.D., Senior Scientist, Rudjer Boskovic Institute, Bijenicka 54, HR-1000 Zagreb, Croatia. e-mail: zarkovic@irb.hr 
Because of complex pathophysiology and uncertain outcome, traumatic brain injuries (TBI) are an important medical problem. Recent study has shown that there are more than 1.5 million TBI patients every year in USA only (RutlandBrown et al. 2006). Because major causes of TBI are traffic accidents and falls, TBI are in particular of high incidence among children and elderly people, who often do not recover well or even die after TBI. Almost all patients with TBI require emergency care, which is followed by hospitalization in every fifth case. In spite of emergency care, mortality of TBI is high, because of limited therapeutic possibilities and the lack of understanding of pathophysiology of TBI, which is associated with local and systemic stress response that affects neuroendocrine and immune system (Schlag et al. 1997; Wildburger et al. 1998) Therefore, intense systemic inflammatory response to TBI which affects both injured and healthy cerebral tissue is often important consequence of TBI (Morganti-Kossmann et al. 2001). Inflammatory reaction involved in the pathogenesis of TBI is essential component of complex systemic stress response which includes the opposing effects of the propagation of secondary brain damage and enhanced repair of injured tissue. Recent research implies that the complete elimination of certain inflammatory mediators might even be detrimental (Stahel et al. 2000) but the precise role of each mediator still remains to be elucidated.

Inflammatory stress response to TBI includes activation of complement (Kaczorowski et al. 1995) and up-regulation of adhesion molecules on the endothelium of cerebral blood vessels (Whalen et al. 1999), associated with the accumulation of neutrophils and production of cytokines (Yamasaki et al. 1997). Interleukin-8 (IL-8) is considered to be one of the pivotal cytokines in the pathophysiology of TBI. It is a 69-79 aminoacid cytokine produced by various cell types including neutrophils, endothelium (Yamasaki et al. 1997), microglia (Ehrlich et al. 1998) and astrocytes (Croitoru-Lamoury 2003). The release of IL-8 is stimulated by other cytokines like interleukin-1 (IL-1) and by hypoxia, ischemia and reperfusion, which make the basis of posttraumatic oxidative stress (Zhang et al. 1999; Wildburger et al. 2000, 2001; Emir et al. 2005; Nikolic-Heitzler et al. 2006). Recently, the synergistic effect on IL-8 secretion of tumor necrosis factor- $\alpha$ (TNF- $\alpha$ ) and soluble intercellular adhesion molecule (sICAM-1) on murine macrophage inflammatory protein (MIP-2) (murine IL-8 analogue) has been implicated (Otto et al. 2000). IL-8 binds to G-protein coupled receptors on neutrophils and stimulates chemotaxis, changes in morphology, adhesion, diapedesis and release of lysosmal enzymes (Hoch et al. 1996). It increases vascular permeability (Smith at al. 1991), and stimulates migration of neutrophils from blood to cerebrospinal fluid in patients with bacterial meningitis and encephalopathy (Mastroianni et al. 1994; Minami et al. 2004). Autoantibodies to IL-8 were found to reduce size of brain tissue damage in an animal model of cerebral ischemia and reperfusion, probably because they inhibit migration of leucocytes in ischemic areas (Stahel et al. 2000). Although numerous experimental data indicate that IL-8 might have a crucial role in the inflammatory stress response to TBI, there are only a few clinical studies on IL-8 in patients after TBI with unequal results (Kossmann et al. 1997; Whalen et al. 2000; Mussack et al. 2002). Therefore, we determined IL-8 levels in central venous blood plasma and cerebrospinal fluid (CSF) of patients with isolated TBI at admittance to Intensive Care Unit and analyzed their potential relevance as prognostic indicators of mortality. The age of patients as well as clinical scoring systems Acute Physiologic and Chronic Health Evaluation (APACHE II) and Glasgow Coma Scale (GCS) known to be reliable predictive parameters in TBI patients (Ghajar 2000; Boto et al. 2006) were also analyzed in respect to the mortality.

\section{Patients and Methods}

\section{Patients}

Twenty adult patients (14 men, 6 women) with isolated severe TBI (GCS $\leqq 8$ ) were included in the study. The average age of patients was 53 years. Exclusion criteria included any injury to other organ systems, history 
of stroke, dementia, autoimmune diseases, acute infection and medication with anti-inflammatory drugs.

Intracranial pressure (ICP) of all patients was constantly monitored at Intensive Care Unit using intraventricular catheter. Jugular bulb catheter was placed to patients with intracranial hypertension (intracranial pressure $>20 \mathrm{mmHg}$ ). Intracranial hypertension was treated using moderate hyperventilation $\left(\mathrm{paCO}_{2}=4.2 \mathrm{kPa}\right)$ and drainage of cerebrospinal fluid, aiming of stabilize cerebral perfusion pressure (mean arterial pressure minus intracranial pressure) of $70 \mathrm{~mm} \mathrm{Hg}$.

All patients had central venous catheter and arterial line and were mechanically ventilated. Corticosteroids were not administrated.

Outcome was defined as intrahospital mortality within 30 days of admittance to Intensive Care Unit. Clinical status of the patients was assessed using standard clinical scoring systems GCS and APACHE II.

The study was approved by the hospital Ethic Committee while informed consent was obtained from the relatives of the patients.

\section{Cerebrospinal fluid and blood samples}

The samples were taken at admittance to the Intensive Care Unit. Blood samples for determination of IL-8 were taken from central venous line and jugular bulb catheter, centrifuged and frozen $-80^{\circ} \mathrm{C}$. The samples of CSF were collected simultaneously and were equally stored at $-80^{\circ} \mathrm{C}$ until analyzed. All standard biochemical and coagulation tests were analyzed from central venous blood sample, while acid-base status and lactate levels were analyzed from arterial line blood.

\section{Determination of interleukin-8}

The values of IL- 8 in plasma and CSF were analyzed by enzyme-linked immunosorbent assay (ELISA). The microplate format IL-8 kit, designed for the quantitative measurement of IL-8 was used according to manufacturer's instructions (Millenia, Biotec GmbH, Bad Neuheim, Germany). Lower limit of sensitivity was 3.5 $\mathrm{pg} / \mathrm{ml}$. Coefficient of variation was below $10 \%$.

\section{Statistical analysis}

The values of IL- 8 were expressed as the mean \pm S.E. Mann Whitney's U-test was applied to compare the respective values of the parameters between survivors and non survivors. Linear regression test was used to analyze correlation of IL- 8 with biochemical and pressure variables. Receiver Operating Characteristics (ROC) was applied to determine the predictive relevance of IL-8 determination.

\section{Results}

While ten patients recovered well, the other ten patients died. As can be seen in Tables 1 and 2, mortality was associated with GCS $(6.0 \pm 0.7$ in survivors, vs $4.1 \pm 0.4$ in nonsurvivors, $p<0.05$ ) and with APACHE II $(14.0 \pm 1.7$ in survivors, vs $19.8 \pm 1.0$ in nonsurvivors, $p<0.01)$. There was no association between mortality and any routine blood parameters analyzed or pressure variables, including intracranial pressure (not presented).

The IL-8 levels determined from central venous blood samples were significantly $(p<$ $0.05)$ lower in survivors $(71.00 \pm 14.17 \mathrm{pg} / \mathrm{ml})$,

TABLE 1. The average values of clinical and biochemical parameters determined for survivors and non-survivors after TBI.

\begin{tabular}{lcc}
\hline \multicolumn{1}{c}{ Parameter } & Survivors & Non survivors \\
\hline GCS $^{*}$ & $6.0 \pm 0.7$ & $4.1 \pm 0.4$ \\
APACHE II $^{* *}$ & $14.0 \pm 1.7$ & $19.8 \pm 1.0$ \\
Age $^{*}$ & $48.6 \pm 6.3$ & $58.7 \pm 4.7$ \\
CSF IL-8 NS & $924.86 \pm 144.67$ & $793.67 \pm 121.67$ \\
Central venous IL-8 $^{*}$ & $71.0 \pm 14.17$ & $111.26 \pm 16.9$ \\
\hline
\end{tabular}

Results are presented as mean \pm S.E.

Significance (Mann-Whitney test) - ${ }^{*} p<0.05 ;{ }^{* *} p<0.01$; NS - not significant $p>0.1$.

APACHE II, Acute Physiologic and Chronic Health Evaluation; GCS, Glasgow Coma Scale; IL-8, interleukin 8; CSF, cerebrospinal fluid. 
TABLE 2. Clinical and biochemical parameters related with mortality.

\begin{tabular}{lccccc}
\hline \multicolumn{1}{c}{ Parameter } & Cutoff value & PPV $(\%)$ & NPV $(\%)$ & Sensitivity (\%) & Specificity (\%) \\
\hline APACHE II & 20 & 77 & 100 & 100 & 70 \\
GCS & 5 & 78 & 73 & 70 & 80 \\
Age & 51 & 67 & 64 & 60 & 70 \\
Central venous IL-8 & $52 \mathrm{pg} / \mathrm{ml}$ & 64 & 83 & 90 & 50 \\
Jugular bulb IL-8 & $39 \mathrm{pg} / \mathrm{ml}$ & 64 & 67 & 90 & 30 \\
\hline
\end{tabular}

PPV, positive predictive value; NPV, negative predictive value; APACHE II, Acute Physiologic and Chronic Health Evaluation; GCS, Glasgow Coma Scale; IL-8, interleukin 8.

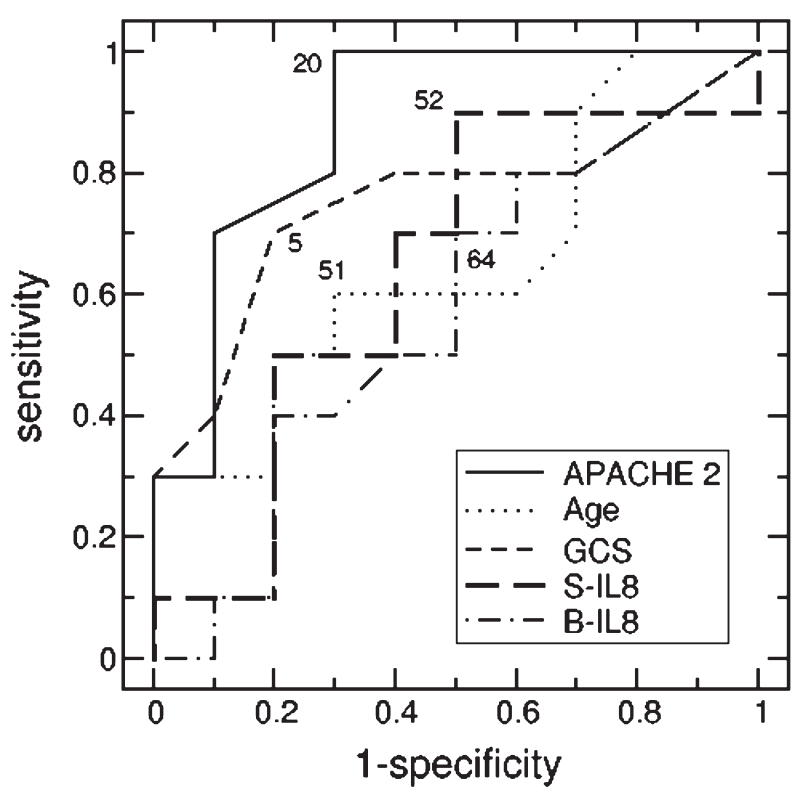

Fig. 1. Receiver operating characteristic curves (ROC) of the relevant prognostic markers in the blood of TBI patients at admittance.

The curves display relationship between sensitivity and 1-specificity and the respective optimal cut off values. The area under curve (AUC) was 0.88 for APACHE II, 0.65 for age, 0.75 for GCS and 0.64 for S IL-8 from central venous (subclavian) sample. Jugular bulb IL-8 (B- IL8) was marginally significant with AUC of 0.57 .

APACHE II, Acute Physiologic and Chronic Health Evaluation; GCS, Glasgow Coma Score; S-IL8, IL-8 values in subclavian venous blood; B-IL8, IL-8 values in jugular bulb blood. than in nonsurvivors $(111.26 \pm 16.9 \mathrm{pg} / \mathrm{ml})$. However, there was no significant difference $(p>$ $0.5)$ between CSF IL-8 levels between survivors $(924.86 \pm 144.67 \mathrm{pg} / \mathrm{ml})$ and nonsurvivors (793.67 $\pm 121.67 \mathrm{pg} / \mathrm{ml})$.

There was no correlation between plasma and CSF IL-8 levels $(r=-0.18)$. We also did not find any relation between plasma or CSF values of IL- 8 with age or gender of our patients. However, the age of survivors was slightly, but significantly $(p<0.05)$ higher in nonsurvivors $(58.7 \pm 4.7)$ than in survivors $(48.6 \pm 6.3)$.

ROC analysis (Fig. 1) revealed significant prognostic value of GCS, APACHE II and IL-8 in central venous sample. Area Under Curve (AUC) to predict mortality was 0.75 for the GCS, 0.88 for the APACHE II, 0.65 for age and 0.64 for central venous IL-8. Jugular bulb IL-8 (B-IL8) was marginally significant with AUC of 0.57 .

\section{Discussion}

Our results suggest that the plasma level of IL-8 could be predictive parameter of mortality in patients with severe TBI, which is in agreement with findings obtained in other studies analyzing association of IL-8 with different severe disorders. Namely, plasma levels of IL- 8 were also found associated with mortality after cardiopulmonary resuscitation (Adrie et al. 2002), with acute respiratory distress syndrome (ARDS) (Imai et al. 2003) and in experimental model of braindead donors (Pratschke et al. 2000). Even postmortem analysis of IL- 8 indicated in one study its association with severe traumatic injury (Mimasaka 2002). 
The source of IL-8 in CSF and in plasma could be central nervous system (CNS) itself as well as inflammatory cells. It seems that high plasma level of IL-8 in patients with TBI originates at least in a part from injured brain tissue (Adrie et al. 2002). Increased permeability of the blood-brain barrier in these patients, contributes further to the elevated plasma level of the cytokine. Catecholamines, which are important factors of systemic stress response to trauma that trigger peripheral IL-8 release were also found elevated in urine of patients with severe head injury (Feldman et al. 1993) indicating their potential role in stimulation of IL-8 production after TBI. High levels of endogenous catecholamines and autonomic deregulation can provoke oscillations in blood pressure with hyper-and hypotensive episodes, which can both compromise peripheral perfusion and contribute to peripheral neutrophil accumulation, adhesion to vascular endothelium and to release of reactive oxygen species. Under such circumstances propagation of oxidative stress and inflammation may occur even if initial injury did not cause instant raise of pro-inflammatory cytokines. In agreement with that are findings of IL- 8 changes in an experimental model of the extreme form of brain injury, where brain-death caused elevation of inflammatory mediators in peripheral organs, while the raise of IL-8 in CSF was noticed also after peripheral trauma that did not affect the CNS directly (Chuang et al. 2005). The raise of IL-8 in CSF can be also found in various neurological syndromes in particular in neurodegenerative diseases (Stoeck et al. 2006). Even in case of moderate brain injury, prompt expression of mRNA of cytokines, chemokines and adhesion molecules was found (Pratschke et al. 2000). It is speculated that these events eventually lead to dysregulation of apoptosis (Papathanassoglou et al. 2001), which plays important role in multiple organ dysfunction syndrome (MODS). In favor of this are experimental findings of effectiveness of antiinflammatory therapy for severe brain injury that was beneficial for cardiac consequences of brain trauma (Emir et al. 2005).

Although it is considered that IL- 8 is one of the crucial mediators of inflammatory response to TBI, the relative prognostic value of IL- 8 in CSF and blood samples should be further studied. In the study conducted by Kossmann et al. (1997), there was no association between mortality and plasma IL-8 concentration. Similar results were published by McKeating et al. (1997) who found that plasma IL-8 level was not elevated in patients with TBI. On the contrary, the recent study by Mussack et al. (2002) revealed that high IL-8 plasma level after TBI may be correlated with unfavorable neurological outcome. In a similar study Kushi et al. (2003) found that IL-8 in peripheral blood was significantly higher in nonsurvivors after $72 \mathrm{hrs}$, but not at admission. IL-8 was not analyzed in the jugular bulb plasma in any of these studies.

The results of our investigation are consistent with the one conducted by Mussack et al. (2002) and imply that IL-8 plasma level could be an early marker of neurological deficit and lethal outcome. Lower predictive values of IL-8 in jugular bulb sample among our patients imply that systemic, rather than cerebral, inflammatory reaction might be responsible for the deterimental effects.

In favor of this assumption is the lack of association of IL-8 level in CSF and mortality. Our results are consistent with another study on adult patients in which authors did not reveal any association of the IL-8 values in CSF with mortality although they found IL-8 values in CSF related to the dysfunction of the blood: brain barrier (Kossmann et al. 1997). Opposite to that in the study involving children with severe head injury, IL-8 concentration in CSF was associated with lethal outcome (Whalen et al. 2000). Kushi et al. (2003) found significantly higher CSF IL-8 than peripheral blood level, but association with lethal outcome was established only after $72 \mathrm{hrs}$. Therefore, it is possible that the raise of IL- 8 in CSF develops after the rapid increase of the blood values of IL- 8 that are due to post-traumatic systemic stress response.

The differences between concentrations of IL-8 in CSF and in plasma could indicate consumption of IL- 8 by the CNS tissues as well as 
intrathecal production of the cytokine. The values of CSF IL-8 among healthy volunteers also support physiological synthesis of IL-8 by the CNS (Maier et al. 2005). Under pathological conditions such as meningitis or astrocytoma development synthesis of IL-8 in the brain is pronounced resulting in the raise of the cytokine values in CSF (Mastroianni et al. 1998; Choi 2002). In the study by Kossmann et al. (1997) cerebrospinal IL-8 level correlated with the concentration of nerve growth factor. Therefore, it is possible that IL-8 is also involved in the regenerative process of inflammatory reaction to TBI. As TNF/ lymphotoxin- $\alpha$ deficient mice have increased posttraumatic mortality, it is implied that proinflammatory cytokines might mediate beneficial effects on brain tissue after TBI (Stahel et al. 2000). If so, IL-8 might have dual activities that would be time-dependent and reflect origin of the cytokine, peripheral IL-8 might reflect harmful posttraumatic systemic stress response, while intrathecal IL-8 production could reflect not only neuroinflammation but also tissue regeneration. This hypothesis should be further studied.

Therefore, the results of our study imply that plasma IL-8 might be useful prognostic indicator in adult patients after TBI, comparative to the well known predictors GCS, APACHE II and age, while further studies are needed to elucidate biomedical aspects of its synthesis and activities.

\section{Acknowledgments}

The study was supported by Croatian Ministry of Science, Education and Sports and by COST Action B35.

\section{References}

Adrie, C., Adib-Conquy, M., Laurent, I., Monchi, M., Vinsonneau, C., Fitting, C., Fraisse, F., Dinh-Xuan, A.T., Carli, P., Spaulding, C., Dhainaut, J.F. \& Cavaillon, J.M. (2002) Successful cardiopulmonary resuscitation after cardiac arrest as a "sepsis-Like" syndrome. Circulation, 106, $562-568$.

Boto, G.R., Gomez, P.A., De la Cruz, J. \& Lobato, R.D. (2006) Severe head injury and the risk of early death. J. Neurol. Neurosurg. Psychiatry, 77, 1054-1059.

Choi, C., Kutsch, O., Park, J., Zhou, T., Seol, D.W. \& Benveniste, E.N. (2002) Tumor necrosis factor-related apoptosis-inducing ligand induces caspase-dependent interleukin- 8 expression and apoptosis in human astroglioma cells. Mol. Cell. Bio., 22, 724-736.
Chuang, D., Power, S.E., Dunbar, P.R. \& Hill, A.G. (2005) Central nervous system interleukin-8 production following neck of femur fracture. ANZ J. Surg., 75, 813-816.

Croitoru-Lamoury, J., Guillemin, G.J., Boussin, F.D., Mognetti, B., Gigout, L.I., Cheet, A., Vaslin, B., Le Grand, R., Brew, B.J. \& Dormont, D. (2003) Expression of chemokines and their receptors in human and simian astrocytes: Evidence for a central role of TNF alpha and IFN gamma in CXCR4 and CCR5 modulation. Glia, 41, 354-370.

Ehrlich, L.C., Hu, S.X., Sheng, W.S., Sutton, R.L., Rockswold, G.L., Peterson, P.K. \& Chao, C.C. (1998) Cytokine regulation of human microglial cell IL-8 production. J. Iтти nol., 160, 1944-1948.

Emir, M., Ozisik, K., Cagli, K., Ozisik, O., Tuncer, S., Bakuy, V., Yildirim, E., Kilinc, K. \& Gol, K. (2005) Beneficial effect of methylprednisolone on cardiac myocytes in a rat model of severe brain injury. Tohoku J. Exp. Med., 207, 119-124.

Feldman, Z., Contant, C.F., Pahwa, R., Goodman, J.C., Robertson, C.S., Narayan, R.K. \& Grossman, R.G. (1993) The relationship between hormonal mediators and systemic hypermetabolism after severe head injury. Trauma, 34, 806-812.

Ghajar, J. (2000) Traumatic brain injury. Lancet, 356, 923-929.

Hoch, R.C., Scraufstatter, I.U. \& Cochrane, C.G. (1996) In vivo, in vitro and molecular aspects of interleukine- 8 and the interleukin-8 receptors. J. Lab. Clin. Med., 128, 134.

Imai, Y., Parodo, J., Kajikawa, O., de Perrot, M., Fischer, S., Edwards, V., Cutz, E., Liu, M., Keshavjee, S., Martin, T., Marshall, J., Ranieri, V.M. \& Arthur, S. (2003) Injurious mechanical ventilation and end-organ epithelial cell apoptosis and organ dysfunction in an experimental model of acute respiratory distress syndrome. JAMA, 9, 2104-2112.

Kaczorowski, S.L., Schiding, J.K., Toth, C.A. \& Kochanek, P.M. (1995) Effect of soluble complement receptor-1 on neutrophil accumulation after traumatic brain injury in rats. $J$. Cereb. Blood Flow Metab., 15, 860-864.

Kossmann, T., Stahel, P.F., Lenzlinger, P.M., Redl, H., Dubs, R.W., Trentz, O., Schlag, G. \& Morganti-Kossmann, M.C. (1997) Interleukin-8 released into the cerebrospinal fluid after brain injury is associated with blood-brain barrier dysfunction and nerve growth factor production. J. Cereb. Blood Flow Metab., 17, 280-289.

Kushi, H., Saito, T., Makino, K. \& Hayashi, N. (2003) IL-8 is a key mediator of neuroinflammation after traumatic brain injury. Crit. Care Med. (Suppl.), 31, 363.

Maier, B., Laurer, H.L., Rose, S., Buurman, W.A. \& Marzi, I. (2005) Physiological levels of pro- and anti-inflammatory mediators in cerebrospinal fluid and plasma: A normative study. J. Neurotrauma, 22, 822-835.

Mastroianni, C.M., Paoletti, F., Rivosecchi, R.M., Lancella, L., Ticca, F., Vullo, V. \& Delia, S. (1994) Cerebrospinal fluid interleukin -8 in children with purulent bacterial and tuberculosus meningitis. Ped. Inf. Dis. J., 13, 1008-1010.

Mastroianni, C.M., Lancella, L., Mengoni, F., Lichtner, M., Santopadre, P., Dagostino, C., Ticca, F. \& Vullo, V. (1998) Chemokine profiles in the cerebrospinal fluid (CSF) during the course of pyogenic and tuberculous meningitis. Clin. Exp. Immunol., 22, 724-736.

McKeating, E.G., Andrews, P.J.D., Signorini, D.F. \& Mascia, L. (1997) Transcranial cytokine gradients in patients requiring intensive care after acute brain injury. Br. J. Anaesth., 78, 520-523.

Mimasaka, S. (2002) Postmortem cytokine levels and the cause of death. Tohoku J. Exp. Med., 197, 145-150. 
Minami, K., Yanagawa, T., Okuda, M., Suzuki, H., Tamura, A., Izumi, G. \& Yoshikawa, N. (2004) Cerebrospinal fluid cytokines in Salmonella Urbana encephalopathy. Tohoku J. Exp. Med., 203, 129-132.

Morganti-Kossmann, M.C., Rancan, M., Otto, V.I., Stahel, P.F. \& Kossmann, T. (2001) Role of cerebral inflammation after traumatic brain injury: A revisited concept. Shock, 16, 165-177.

Mussack, T., Biberthaler, P., Kanz, K.G., Wiedermann, E., Gippner-Steppert, C., Mutscler, W. \& Jochum, M. (2002) Serum S-100 B and interleukin- 8 as predictive markers for comparative neurologic outcome analysis of patients after cardiac arrest and severe traumatic brain injury. Crit. Care. Med., 30, 2669-2674.

Nikolic-Heitzler, V., Rabuzin, F., Tatzber, F., Vrkic, N., Bulj, N., Borovic, S., Wonisch, W., Mazul-Sunko, B. \& Zarkovic, N. (2006) Persistent oxidative stress after myocardial infarction treated by percutaneous coronary intervention. Tohoku J. Exp. Med., 210, 247-255.

Otto, V.I., Heinzel-Pleines, U.E., Gloor, S.M., Trentz, O., Kossmann, T. \& Morganti-Kossmann, M.C. (2000) sICAM-1 and TNF-alpha induce MIP-2 with distinct kinetics in astrocytes and brain microvascular endothelial cells. J. Neurosci. Res., 60, 733-742.

Papathanassoglou, E.D.E., Moynihan, J.A. \& Ackerman, M.H. (2001) Does programmed cell death (apoptosis) play a role in the development of multiple organ dysfunction in critically ill patients? Crit. Care. Med., 28, 537-549.

Pratschke, J., Wilhelm, M.J., Kusaka, M., Beato, F., Milford, E.L., Hancock, W.W. \& Tilney, N.L. (2000) Accelerated rejection of renal allografts from brain-dead donors. Ann. Surg., 232, 263-271.

Rutland-Brown, W., Langlois, J.A., Thomas, K.E. \& Lily, Y.L. (2006) Incidence of traumatic brain injury in the United States, 2003. J. Head Trauma Rehab., 21, 544-548.

Schlag, G., Zarkovic, K., Red, H., Zarkovic, N. \& Waeg, G. (1997) Brain damage secondary to hemorrhagic shock in baboons. In: Shock, Sepsis and Organ Failure, 5th Wiggers Bernard Conference, edited by G. Schlag, H. Redl \& D.L. Traber Springer-Verlag, Heidelberg, pp. 3-17.

Smith, W.B., Gamble, J.R, Clark-Lewis, I. \& Vadas, M.A. (1991) Interleukin-8 induces neutrophil transendothelial migration. Immunology, 72, 65-72.

Stahel, P.F., Shohami, E., Younis, F.M., Kariya, K., Otto, V.I., Lenzlinger, P.M., Grosjean, M.B., Eugster, H.P., Trentz, O.,
Kossmann, T. \& Morganti-Kossmann, M.C. (2000) Experimental closed head injury: Analysis of neurological outcome, blood-brain barrier dysfunction, intracranial neutrophil infiltration, and neuronal cell death in mice deficient in genes for pro-inflammatory cytokines. J. Cereb. Blood Flow Metab., 20, 369-380.

Stoeck, K., Bodemer, M. \& Zerr, I. (2006) Pro- and antiinflammatory cytokines in the CSF of patients with Creutzfeldt-Jakob disease. Neuroimmunology, 172, 175-181.

Whalen, M.J., Carlos, T.M., Dixon, C.E., Schiding, J.K., Clark, R.S.B., Baum, E., Yan, H.Q., Marion, D.W. \& Kochanek, P.M. (1999) Effect of traumatic brain injury in mice deficient in intercellular adhesion molecule-1: Assessment of histopathologic and functional outcome. J. Neurotrauma, 16, 299-309.

Whalen, M.J., Carlos, T.M., Kochanek, P.M., Wisniewski, S.R. Bell, M.J., Clark, R.S.B., DeKosky, S.T., Marion, D.W. \& Adelson, P.D. (2000) Interleukin-8 is increased in cerebrospinal fluid of children with severe head injury. Crit. Care Med., 28, 929-934.

Wildburger, R., Zarkovic, N., Tonkovic, G., Skoric, T., Frech, S., Hartleb, M., Loncaric, I. \& Zarkovic, K. (1998) Posttraumatic hormonal disturbances: Prolactin as a link between head injury and enhanced osteogenesis. J. Endrocrinol. Invest., 21, 78-86.

Wildburger, R., Borovic, S., Zarkovic, N. \& Tatzber, F. (2000) Post-traumatic dynamic changes of the antibody titer against oxidized low density lipoproteins. Wien. Klin. Wochenschr., 112, 798-803.

Wildburger, R., Zarkovic, N., Leb, G., Borovic, S., Zarkovic, K. $\&$ Tatzber, F. (2001) Post-traumatic changes of insulin like growth factor type 1 and growth hormone in patients with bone fractures and traumatic brain injury. Wien. Klin. Wochenschr., 113, 119-126.

Yamasaki, Y., Matsuo, Y., Zagorski, J., Matsuura, N., Onodera, H., Itoyama, Y. \& Kogure, K. (1997) New therapeutic possibilities of blocking cytokine - induced neutrophil chemotractant on transient ischemic brain damage in rats. Brain Res., 759, 103-111.

Zhang, W.D., Smith, C., Shapiro, A., Monette, R., Hutchison, J. \& Stanimirovic, D. (1999) Increased expression of bioactive chemokines in human cerebromicrovascular endothelial cells and astrocytes subjected to simulated ischemia in vitro. J. Neuroimmunol., 101, 148-160. 